\title{
Rényi entropies and nonlinear diffusion equations
}

\author{
G. Toscani*
}

February 6, 2020

\begin{abstract}
Since their introduction in the early sixties [20, the Rényi entropies have been used in many contexts, ranging from information theory to astrophysics, turbulence phenomena and others. In this note, we enlighten the main connections between Rényi entropies and nonlinear diffusion equations. In particular, it is shown that these relationships allow to prove various functional inequalities in sharp form.
\end{abstract}

Keywords: Rényi entropy, Nonlinear diffusion equations, Sobolev type inequalities.

\section{Introduction}

Given a probability density $f(x), x \in \mathbb{R}^{n}$, and a positive constant $p$ the Rényi entropy of order $p$ of $f$ is defined by [14]:

$$
\mathcal{R}_{p}(f)=\frac{1}{1-p} \log \left(\int_{\mathbb{R}^{n}} f^{p}(y) d y\right) .
$$

This concept of entropy has been introduced by Rényi in [20] for a discrete probability measure to generalize the classical logarithmic entropy, by maintaining at the same time most of its properties. Indeed, the Rényi entropy of order 1, defined as the limit as $p \rightarrow 1$ of $\mathcal{R}_{p}(f)$ is

$$
\lim _{p \rightarrow 1} \mathcal{R}_{p}(f)=\mathcal{R}(u)=-\int_{\mathbb{R}^{n}} f(y) \log f(y) d y .
$$

Therefore, the standard (Shannon) entropy of a probability density [22] is included in the set of Rényi entropies, and it is identified with the Rényi entropy of index $p=1$.

Among other properties, the Rényi entropy (11) behaves as the Shannon entropy (2) with respect to the scaling for dilation of the probability density. As usual, for any given density $f(x)$ and positive constant $a$, we define the dilation of $f$ by $a$, as the masspreserving scaling

$$
f(x) \rightarrow f_{a}(x)=a^{n} f(a x) .
$$

\footnotetext{
*Department of Mathematics, University of Pavia, via Ferrata 1, 27100 Pavia, ITALY.
} 
Then, for any $p \geq 0$ it holds

$$
\mathcal{R}_{p}\left(f_{a}\right)=\mathcal{R}_{p}(f)-n \log a .
$$

This characteristic differentiates the Rényi entropy from other generalizations of the Shannon entropy, which have been introduced later on on the literature. For example, the Tsallis entropy of order $p$ [25]:

$$
\mathcal{T}_{p}(f)=\frac{1}{1-p} \int_{\mathbb{R}^{n}}\left(f^{p}(y)-f(y)\right) d y,
$$

which is extensively used by physicists in statistical mechanics [26], does not satisfy property (4). As we shall see in Section 2, property (4) is one of the main ingredients to work with the Rényi entropy functional and to derive from it inequalities in sharp form. Thus, in our opinion, the definition (11) introduced by Rényi represents a very coherent generalization of the Shannon entropy.

The Shannon entropy is naturally coupled to the heat equation (with diffusion coefficient $\kappa$ ) posed in the whole $\mathbb{R}^{n}$

$$
\frac{\partial u(x, t)}{\partial t}=\kappa \Delta u(x, t)
$$

as soon as the initial datum given is assumed to be a probability density. As recently noticed in [28], the deep link between the Shannon entropy and the heat equation started to be used as a powerful instrument to obtain mathematical inequalities in sharp form in the years between the late fifties to mid sixties. To our knowledge, the first application of this idea can be found in two papers by Linnik [16] and Stam [23] (cf. also Blachman [4]), published in the same year and concerned with two apparently disconnected arguments. Stam [23] was motivated by the finding of a rigorous proof of Shannon's entropy power inequality [22], while Linnik [16] used the information measures of Shannon and Fisher in a proof of the central limit theorem of probability theory. Also, in the same years, the heat equation has been used in the context of kinetic theory of rarefied gases by McKean [15] to investigate that large-time behaviour of Kac caricature of a Maxwell gas. There, various monotonicity properties of the derivatives of the Shannon entropy along the solution to the heat equation have been derived.

Likewise, the Rényi entropy od order $p$ is strongly coupled to the nonlinear diffusion equation of order $p$ posed in the whole $\mathbb{R}^{n}$

$$
\frac{\partial v(x, t)}{\partial t}=\kappa \Delta v^{p}(x, t)
$$

still with the initial datum which is assumed to be a probability density. This maybe not so well-known link has been outlined in some recent papers [, 21, where various results valid for the Shannon entropy have been shown to hold also for the Rényi entropies, and applied to the study of the large-time behavior of the solution to equation (7). We aim in this note to highlight this connection. 


\section{Nonlinear diffusion equations and self-similarity}

To start with, let us recall briefly some essential features of the nonlinear diffusion equation (7). Existence an uniqueness of the solution of (7) to the initial value problem posed in the whole space is well-known [5, 29], and we address the interested reader to these references for details. The forthcoming analysis will be restricted to initial data which are probability densities with finite variance, and it will include both the case $p>1$, usually known as porous medium equation, and the case $p<1$, the fast diffusion equation. In dimension $n \geq 1$, the range of exponents which ensure the boundedness of the second moment of the solution is $p>\bar{p}$ with $\bar{p}=n /(n+2)$, which contains a part of the so-called fast diffusion range $p<1$. The particular subinterval of $p$ is motivated by the existence of a precise solution, found by Zel'dovich, Kompaneets and Barenblatt in the fifties (briefly called here Barenblatt solution) [2, 3, 31, which serves as a model for the asymptotic behavior of a wide class of solutions with finite second moment. In the case $p>1$ (see [5] for $p<1$ ) the Barenblatt (also called self-similar or generalized Gaussian solution) departing from $x=0$ takes the self-similar form

$$
M_{p}(x, t):=\frac{1}{t^{n / \mu}} \tilde{M}_{p}\left(\frac{x}{t^{1 / \mu}}\right)
$$

where

$$
\mu=2+n(p-1)
$$

and $\tilde{M}_{p}(x)$ is the time-independent function

$$
\tilde{M}_{p}(x)=\left(C-\lambda|x|^{2}\right)_{+}^{\frac{1}{p-1}} .
$$

In (91) $(s)_{+}=\max \{s, 0\}, \lambda=\frac{1}{2 \mu} \frac{p-1}{p}$, and the constant $C$ can be chosen to fix the mass of the source-type Barenblatt solution equal to one.

The solution to equation (7) satisfies mass and momentum conservations, so that

$$
\int_{\mathbb{R}^{n}} v(x, t) d x=1 ; \quad \int_{\mathbb{R}^{n}} x v(x, t) d x=0 ; \quad t \geq 0 .
$$

Hence, without loss of generality, one can always assume that $v_{0}(x)$ is a probability density of first moment equal to zero. Let us define by $E(v(t))$ the second moment of the solution:

$$
E(v(t))=\int_{\mathbb{R}^{n}}|x|^{2} v(x, t) d x .
$$

Then, $E(v(t))$ increases in time from $E_{0}=E\left(v_{0}\right)$, and its evolution is given by the nonlinear law

$$
\frac{d E(v(t))}{d t}=2 n \int_{\mathbb{R}^{n}} v^{p}(x, t) d x \geq 0,
$$


which is not explicitly integrable unless $p=1$. The second moment of the solution to equation (7) has an important role in connection with the knowledge of the large time behavior of the solution. Also, in presence of a finite second moment we can immediately establish a deep connection between equation (7) and the Rényi entropy of the same order $p$.

Indeed, let us consider the evolution in time of the Rényi entropy of order $p$ along the solution of the nonlinear diffusion equation (7). Integration by parts immediately yields

$$
\frac{d}{d t} \mathcal{R}_{p}(v(\cdot, t))=\mathcal{I}_{p}(v(\cdot, t)), \quad t>0,
$$

where, for a given probability density $f(x)$

$$
\mathcal{I}_{p}(f):=\frac{1}{\int_{\mathbb{R}^{n}} f^{p} d x} \int_{\{f>0\}} \frac{\left|\nabla f^{p}(x)\right|^{2}}{f(x)} d x .
$$

When $p \rightarrow 1$, identity (13) reduces to DeBruijn's identity, which connects Shannon's entropy functional with the Fisher information

$$
\mathcal{I}(f)=\int_{\{f>0\}} \frac{|\nabla f|^{2}}{f} d y .
$$

via the heat equation [4, 11, 23. Since $\mathcal{I}_{p}(f)>0$, identity (13) shows that the Rényi entropy of the solution to equation (77) is increasing in time.

Since the energy scales under the dilation (4) of $f$ according to

$$
E\left(f_{a}\right)=\int_{\mathbb{R}^{n}}|v|^{2} f_{a}(v) d v=\frac{1}{a^{2}} E(f),
$$

if the probability density has bounded second moment, a dilation invariant functional is obtained by coupling Rényi entropy of $f$ with the logarithm of the second moment of $f$

$$
\Lambda_{p}(f)=\mathcal{R}_{p}(f)-\frac{n}{2} \log E(f) .
$$

Let $v(x, t)$ be a solution to equation (7). If we now compute the time derivative of $\Lambda_{p}(v(t))$, we obtain

$$
\frac{d}{d t} \Lambda_{p}(v(t))=\mathcal{I}_{p}(v(t))-n^{2} \frac{\int_{\mathbb{R}^{n}} v^{p}(t) d x}{E(v(t))},
$$

which is a direct consequence of both identities (12) and (13).

The right-hand side of (18) is nonnegative. This can be easily shown by an argument which is often used in this type of proofs, and goes back at least to McKean [15]. One obtains

$$
0 \leq \int_{\{v>0\}}\left(\frac{\nabla v^{p}(x)}{v(x)}+n x \frac{\int v^{p}(x)}{E(v)}\right)^{2} \frac{v(x)}{\int v^{p}} d x=
$$




$$
\begin{gathered}
\mathcal{I}_{p}(v)+n^{2} \frac{\int v^{p}}{E(v)^{2}} \int_{\mathbb{R}^{n}}|x|^{2} v(x) d x+2 n \frac{\int v^{p}}{E(v)} \int_{\{v>0\}} x \cdot \nabla v(x) d x= \\
\mathcal{I}_{p}(v)+n^{2} \frac{\int v^{p}}{E(v)}-2 n^{2} \frac{\int v^{p}}{E(v)}=\mathcal{I}_{p}(v)-n^{2} \frac{\int v^{p}}{E(v)} .
\end{gathered}
$$

Note that equality to zero in (19) holds if and only if, when $v(x)>0$

$$
\frac{\nabla v^{p}(x)}{v(x)}+n x \frac{\int v^{p}}{E(v)}=0
$$

This condition can be rewritten as

$$
\nabla\left(v^{p-1}+\frac{p-1}{2 p}|x|^{2} \frac{n \int v^{p}}{E(v)}\right)=0
$$

which identifies the probability density $v(x)$ as a Barenblatt density in $\mathbb{R}^{n}$ (cf. equation (9)). Also, (19) shows that, among all densities with the same second moment, Fisher information of order $p$ takes its minimum value in correspondence to a Barenblatt density.

We proved that the functional (17) is monotonically increasing in time along the solution to the nonlinear diffusion. The dilation invariance can now be used to identify the limit value. The computation of the limit value uses in a substantial way the scaling invariance property. Indeed, it is well-known that the solution to equation (7) converges towards the self-similar Barenblatt solution (9) in $L_{1}\left(\mathbb{R}^{n}\right)$ at an explicitly computable rate [5, 6, 7, 13. By definition, the second moment of the self-similar solution increases in time, and it is infinite as time goes to infinity. However, by dilation invariance, the value of the functional (17) in correspondence to a Barenblatt function does not depend on its second moment. In other words, we can scale at each time, without changing the value of the functional, in such a way to fix a certain value of the second moment of the Barenblatt when time goes to infinity [27, 28].

The argument we presented is twofold. From one side, it represents a notable tool to study the large-time behavior of solutions to nonlinear diffusion equations. From the other side, it allows to find inequalities by means of solutions to these nonlinear diffusions. Indeed, we proved that, for any probability density function $f$ with bounded second moment

$$
\mathcal{R}_{p}(f)-\frac{n}{2} \log E(f) \leq \mathcal{R}_{p}\left(B_{p, \sigma}\right)-\frac{n}{2} \log E\left(B_{p, \sigma}\right),
$$

where, for $\sigma>0$, we denoted by $B_{p, \sigma}(x)$ the Barenblatt density defined in (8), of second moment equal to $\sigma$. Clearly (21) implies that, under a variance constraint, the Rényi entropy power of order $p$ is maximized by a Barenblatt type density.

Inequality (21) can be rephrased in a slightly different way. Let $f(x)$ be a probability density function in $\mathbb{R}^{n}$, and let $\mathcal{N}_{p}(f)$ denote the entropy power of $f$ associated to the Rényi entropy of order $p$ :

$$
\mathcal{N}_{p}(f)=\exp \left\{\left(\frac{2}{n}+p-1\right) \mathcal{R}_{p}(f)\right\} .
$$


Then, if $p>n /(n+2)$,

$$
\frac{\mathcal{N}_{p}(f)}{E(f)^{1+n(p-1) / 2}} \leq \frac{\mathcal{N}_{p}\left(B_{p, \sigma}\right)}{E\left(B_{p, \sigma}\right)^{1+n(p-1) / 2}}
$$

We note that the definition (22) of $p$-Rényi entropy power, proposed recently in [21, coincides with the classical definition of Shannon entropy power [22], valid when $p=1$. This definition requires $p>(n-2) / n$, in which case $2 / n+p-1>0$. The range of the parameter $p$ for which we can introduce our notion of Rényi entropy power, coincides with the range for which there is mass conservation for the solution of (77) [5]. This range includes the cases in which the Barenblatt has bounded second moment, since $(n-2) / n<$ $n /(n+2)$. We observe that inequality (23) has been derived by a completely different method in [12, 17, 18].

\section{The concavity of Rényi entropy power}

The Shannon entropy power of a probability density,

$$
\mathcal{N}(f)=\exp \left\{\frac{2}{n} \mathcal{R}(f)\right\}
$$

where $\mathcal{R}(f)$, the Shannon entropy, has been defined in (2), is one of the most important concepts of information theory [11]. Starting on it, Shannon derived the celebrated entropy power inequality [4, 23], which reads

$$
\mathcal{N}(f * g) \geq \mathcal{N}(f)+\mathcal{N}(g) .
$$

In (25), $f$ and $g$ are two probability density functions, and $f * g$ denotes convolution. Equality in (25) holds if and only if both $f$ and $g$ are Gaussian densities.

Also, other properties of Shannon entropy power $\mathcal{N}(f)$ have been discovered. In particular, the concavity of entropy power, which asserts that, if $u(x, t)$ is a solution to the heat equation (6) , corresponding to an initial datum $u_{0}(x)$ that is a probability density, then

$$
\frac{d^{2}}{d t^{2}} \mathcal{R}(u(\cdot, t)) \leq 0
$$

Moreover, equality in (26) holds if and only if $u(x, t)$ coincides with the Gaussian density of variance $t$, namely the self-similar solution to the heat equation. Inequality (26) is due to Costa [10. More recently, a short and clean proof of (26) has been obtained by Villani [30], by means of an argument introduced by McKean [15] in his paper on Kac caricature of a Maxwell gas. Recently [27, we investigated various not so well-known consequences of the concavity property (26). Among others, concavity implies the so-called isoperimetric 
inequality for entropies [14]. For any probability density $f(x)$ in $\mathbb{R}^{n}$, this inequality asserts that

$$
\mathcal{N}(f) \mathcal{I}(f) \geq \mathcal{N}\left(M_{\sigma}\right) \mathcal{I}\left(M_{\sigma}\right)
$$

where $\mathcal{I}(f)$ is the Fisher information of $f$ defined in (15) and, for any constant $\sigma>0, M_{\sigma}$ denotes the gaussian density in $\mathbb{R}^{n}$ of mean zero, and variance $\sigma$, that is

$$
M_{\sigma}(x)=\frac{1}{(2 \pi \sigma)^{n / 2}} \exp \left\{-\frac{|x|^{2}}{2 \sigma}\right\} .
$$

As for (21), inequality (31) implies that, the product of the Shannon entropy power and the Fisher information is minimized by a Gaussian density.

The physical idea behind the concavity of the Shannon entropy power is clear. If we evaluate the entropy power in correspondence to a Gaussian density like (28), we obtain

$$
\mathcal{N}\left(M_{\sigma}\right)=2 \pi \sigma e .
$$

Hence, the entropy power of the self similar solution to the heat equation, namely a Gaussian density of variance $2 t$, is a linear function of time, and its second derivative (with respect to time) is equal to zero. This property is restricted to Gaussian densities. Any other solution to the heat equation, different from the self-similar one, is such that its entropy power is concave.

Having in mind to extend the concavity property to the Rényi entropy power, and making use of the result of Section 2, in which we established a connection of the Rényi entropy with the solution of the nonlinear diffusion equation, the starting point for the proof of such a property would be a definition of Rényi entropy power (of order $p$ ) which is consistent with the fact that, when evaluated in correspondence to the Barenblatt selfsimilar solution (8) to the nonlinear diffusion of order $p$, the value of the Rényi entropy power is linear with respect to $t$. It is a simple exercise to verify that, owing to definition (22), this is true, since

$$
\mathcal{N}_{p}\left(M_{p}(t)\right)=\mathcal{N}_{p}\left(\tilde{M}_{p}\right) \cdot t .
$$

In [21], starting from definition (22), we proved that the Rényi entropy power of order $p$ has the concavity property when evaluated along the solution to the nonlinear diffusion (77). The precise result is the following:

Theorem 3.1 ([21] Let $p>(n-2) / n$ and let $u(\cdot, t)$ be probability densities in $\mathbb{R}^{n}$ solving (7) for $t>0$. Then the $p$-th Rényi entropy power defined by (22) satisfies

$$
\frac{d^{2}}{d t^{2}} \mathcal{N}_{p}(v(\cdot, t)) \leq 0
$$

Like in the Shannon's case, inequality (30) lieds to sharp isoperimetric inequalities. The (isoperimetric) inequality for the $p$-th Rényi entropy is contained into the following 
Theorem 3.2 ([21]) If $p>n /(n+2)$ every smooth, strictly positive and rapidly decaying probability density $f$ satisfies

$$
\mathcal{N}_{p}(f) \mathcal{I}_{p}(f) \geq \mathcal{N}_{p}\left(\tilde{M}_{p}\right) \mathcal{I}_{p}\left(\tilde{M}_{p}\right)=\gamma_{n, p} .
$$

We remark that $\mathcal{I}_{p}(f)$ is the generalized Fisher information defined in (14). Once again, it is immediate to show that the product in (31) is invariant under dilation, which allows to reckon explicitly the value of the constant by using the same argument of Section 2. If $p>1$ the value of the constant $\gamma_{n, p}$ is

$$
\gamma_{n, p}=n \pi \frac{2 p}{p-1}\left(\frac{\Gamma\left(\frac{p+1}{p}\right)}{\Gamma\left(\frac{n}{2}+\frac{p+1}{p}\right)}\right)^{2 / n}\left(\frac{(n+2) p-n}{2 p}\right)^{\frac{2+n(p-1)}{n(p-1)}}
$$

In the remaining set of the parameter $p$, that is if $n /(n+2)<p<1$,

$$
\gamma_{n, p}=n \pi \frac{2 p}{1-p}\left(\frac{\Gamma\left(\frac{1}{1-p}-\frac{n}{2}\right)}{\Gamma\left(\frac{1}{1-p}\right)}\right)^{2 / n}\left(\frac{(n+2) p-n}{2 p}\right)^{\frac{2+n(p-1)}{n(p-1)}}
$$

Inequality (31) can be rewritten in a form more suitable to functional analysis. Let $f(x)$ be a probability density in $\mathbb{R}^{n}$. Then, if $p>n /(n+2)$

$$
\int_{\mathbb{R}^{n}} \frac{\left|\nabla f^{p}(x)\right|^{2}}{f(x)} d x \geq \gamma_{n, p}\left(\int_{\mathbb{R}^{n}} f^{p}(x) d x\right)^{\frac{2+2 n(p-1)}{n(p-1)}} .
$$

If $n>2$, the case $p=(n-1) / n$ is distinguished from the others, since it leads to

$$
\frac{2+2 n(p-1)}{n(p-1)}=0, \quad \nu=\frac{1}{n}
$$

and

$$
\mathcal{N}_{1-1 / n}(f)=\int_{\mathbb{R}^{n}} f^{1-1 / n}(x) d x .
$$

In this case the concavity of $\mathcal{N}_{1-1 / n}$ along (7) has been already known and has a nice geometric interpretation in terms of transport distances, see [19].

Note that the restriction $n>2$ implies $(n-1) / n>n /(n+2)$. Hence, for $p=(n-1) / n$ we obtain that the probability density $f$ satisfies the inequality

$$
\int_{\mathbb{R}^{n}} \frac{\left|\nabla f^{(n-1) / n}(x)\right|^{2}}{f(x)} d x \geq \gamma_{n,(n-1) / n} .
$$


The substitution $f=g^{2^{*}}$, where $2^{*}=2 n /(n-2)$, yields

$$
\int_{\mathbb{R}^{n}} \frac{\left|\nabla f^{(n-1) / n}(x)\right|^{2}}{f(x)} d x=\left(\frac{2 n-2}{n-2}\right)^{2} \int_{\mathbb{R}^{n}}|\nabla g(x)|^{2} d x .
$$

Therefore, for any given function $g \geq 0$ such that $g(x)^{2^{*}}$ is a probability density in $\mathbb{R}^{n}$, with $n>2$, we obtain the inequality

$$
\int_{\mathbb{R}^{n}}|\nabla g(x)|^{2} d x \geq\left(\frac{n-2}{2 n-2}\right)^{2} \gamma_{n,(n-1) / n} .
$$

Since

$$
\gamma_{n,(n-1) / n}=n \pi \frac{2^{2}(n-1)^{2}}{n-2}\left(\frac{\Gamma(n / 2)}{\Gamma(n)}\right)^{2 / n},
$$

a simple scaling argument finally shows that, if $g(x)^{2^{*}}$ has a mass different from $1, g$ satisfies the Sobolev inequality [1], 24]

$$
\int_{\mathbb{R}^{n}}|\nabla g(x)|^{2} d x \geq \mathcal{S}_{n}\left(\int_{\mathbb{R}^{n}} g(x)^{2^{*}} d x\right)^{2 / 2^{*}},
$$

where

$$
\mathcal{S}_{n}=n(n-2) \pi\left(\frac{\Gamma(n / 2)}{\Gamma(n)}\right)^{2 / n}
$$

is the sharp Sobolev constant. Hence, Sobolev inequality with the sharp constant is a consequence of the concavity of Rényi entropy power of parameter $p=(n-1) / n$, when $n>2$.

In all the other cases, the concavity of Rényi entropy power leads to GagliardoNirenberg type inequalities with sharp constants, like the ones recently studied by Del Pino and Dolbeault [13, and Cordero-Erausquin, Nazaret, and Villani, [9] with different methods.

\section{Conclusions}

In this note we presented recent results which highlight the links between the Rényi entropies and the nonlinear diffusion equations. This connection is at the basis of an information-theoretic proof of various functional inequalities, which, at difference with previous approaches, have the merit to be intuitive. The main example is furnished by the result of concavity presented in Section 3, where the classical sharp Sobolev inequality is derived as a particular case of the concavity property of the Rényi entropy of order $1-1 / n$. Also, the inequalities presented here can be fruitfully employed to deeply investigate the large-time behavior of the solution to nonlinear diffusion equations [8]. Other 
problems remain to be investigated. Mainly, it would be important to understand if the Shannon entropy power (25), which deals with convolutions, admits a version in terms of Rényi entropy power, whose proof is based on this connection with nonlinear diffusion equations.

\section{References}

[1] T. Aubin, Problémes isopérimétriques et espaces de Sobolev. J. Differential Geometry 11, (1976) 573-598.

[2] G. I. Barenblatt, On some unsteady motions of a liquid and gas in a porous medium. Akad. Nauk SSSR. Prikl. Mat. Meh., 16 (1952) 67-78.

[3] G.I. Barenblatt, Scaling. Self-Similarity and Intermediate Asymptotics. Cambridge Univ. Press, Cambridge 1996.

[4] N.M. Blachman, The convolution inequality for entropy powers. IEEE Trans. Inform. Theory, 11 (1965) 267-271.

[5] A. Blanchet, M. Bonforte, J. Dolbeault, G. Grillo and J. L Vázquez, Asymptotics of the Fast Diffusion Equation via Entropy Estimates. Arch. Ration. Mech. Anal., 191 (2009) 347-385.

[6] M. Bonforte, J. Dolbeault, G. Grillo, and J.-L. Vázquez, Sharp rates of decay of solutions to the nonlinear fast diffusion equation via functional inequalities. Proc. Natl. Acad. Sci. USA, 107 (2010) 16459-16464.

[7] J. A. Carrillo, G. Toscani, Asymptotic $L^{1}$-decay of solutions of the porous medium equation to self-similarity. Indiana Univ. Math. J., 49 (2000) 113-141.

[8] J.A. Carrillo and G. Toscani, Rényi entropy and improved rate of convergence to selfsimilarity for nonlinear diffusion equations. (preprint) (2013).

[9] D. Cordero-Erausquin, B. Nazaret, and C. Villani, A mass-transportation approach to sharp Sobolev and Gagliardo-Nirenberg inequalities. Advances in Mathematics, 182 (2004) 307-332.

[10] M. Costa, A new entropy power inequality. IEEE Trans. Inform. Theory, 31 (1985) 751-760.

[11] T.M. Cover and J.A. Thomas, Elements of Information Theory. Wiley \& Sons, New York, 1992. 
[12] J. Costa, A. Hero and C. Vignat, On solutions to multivariate maximum alphaentropy problems. Lecture Notes in Computer Science, 2683, no. EMMCVPR 2003, Lisbon, 7-9 July 2003, 211-228, 2003.

[13] M. Del Pino, and J. Dolbeault, Best constants for Gagliardo-Nirenberg inequalities and application to nonlinear diffusions. J. Math. Pures Appl., 81 (2002) 847-875.

[14] A. Dembo, T. Cover, and J. Thomas, Information theoretic inequalities. IEEE Trans. Inform. Theory, 37 (1991) 1501-1518.

[15] H.P. McKean JR., Speed of approach to equilibrium for Kacs caricature of a Maxwellian gas. Arch. Rat. Mech. Anal., 21 (1966) 343-367.

[16] Yu.V. Linnik, An information-theoretic proof of the central limit theorem with the Lindeberg condition. Theory Probab. Appl., 4 (1959) 288-299.

[17] E. Lutwak, D. Yang, and G. Zhang, Cramér-Rao and moment-entropy inequalities for Rényi entropy and generalized Fisher information. IEEE Trans. Inform. Theory, $\mathbf{5 1}$ (2005) 473-478.

[18] E. Lutwak, D. Yang and G. Zhang, Moment-entropy inequalities for a random vector. IEEE Trans. Inform. Theory, 53 (2007) 1603-1607.

[19] F. Otto, The geometry of dissipative evolution equations: the porous medium equation. Commun. Part. Diff. Eq., 26 (2001) 101-174.

[20] A. Rényi, On measures of entropy and information. Proc. Fourth Berkeley Symp. Math. Statist. Prob. 1, 547-561. University of California Press, Berkeley 1961.

[21] G. Savaré, and G. Toscani, The concavity of renyi entropy power, IEEE Trans. Inform. Theory, (forthcoming) (2013)

[22] C.E. Shannon, A mathematical theory of communication. Bell Syst. Tech. J. 27 Jul.(1948), 379-423; Oct. (1948) 623-656.

[23] A.J. Stam, Some inequalities satisfied by the quantities of information of Fisher and Shannon. Inf. Contr., 2 (1959) 101-112.

[24] G. Talenti, Best constant in Sobolev inequality. Ann. Mat. Pura Appl., 110 (1976) 353-372.

[25] C. Tsallis, Possible generalization of Boltzmann-Gibbs statistics. J. Stat. Phys., 52 (1988), 479-487.

[26] C. Tsallis, Introduction to Nonextensive Statistical Mechanics - Approaching a Complex World. Springer, New York (2009). 
[27] G. Toscani, An information-theoretic proof of Nash's inequality. Rend. Lincei Mat. Appl., 24 (2013) 83-93.

[28] G. Toscani, Lyapunov functionals for the heat equation and sharp inequalities. Atti Acc. Peloritana Pericolanti, Classe Sc. Fis. Mat. e Nat., 91 (2013) 1-10.

[29] J.L. Vazquez, The Porous Medium Equation: Mathematical Theory, Oxford University Press, Oxford 2007.

[30] C. Villani, A Short Proof of the Concavity of Entropy Power. IEEE Trans. Inform. Theory, 46 (2000) 1695-1696.

[31] Ya.B. Zeldovich, and A.S. Kompaneetz, Towards a theory of heat conduction with thermal conductivity depending on the temperature. Collection of Papers Dedicated to 70th Birthday of Academician A. F. Ioffe, Izd. Akad. Nauk SSSR, Moscow, (1950) 61-71. 